\title{
DESIGUALDADES INJUSTAS: O CONTRADIREITO À SAÚDE*
}

UNFAIR INEQUALITIES: THE HEALTH CARE COUNTER RIGHT

\author{
Sonia Fleury \\ Fundação Getúlio Vargas, Rio de Janeiro, Brasil
}

\begin{abstract}
Resumo
Na Constituição Federal de 1988, foi assegurado o direito universal à Saúde e criado o Sistema Único de SaúdeSUS. Convivemos hoje com uma avançada construção legal que assegura o bem-estar da população por meio de políticas universais, ao lado de uma institucionalidade precária. O objetivo deste trabalho é analisar os dados de pesquisa realizada em hospitais públicos do Rio de Janeiro que utilizou diferentes técnicas qualitativas a fim de identificar os fatores percebidos como condicionadores de desigualdades injustas no acesso e utilização dos serviços de saúde. Os resultados apontam a precariedade material das condições de atendimento, associadas a situações de discriminação social e a práticas de uso de relações pessoais para ter acesso aos serviços públicos, como formas de materialização do contradireito à saúde.
\end{abstract}

Palavras-chave: direito à saúde; desigualdades injustas; discriminação social.

\begin{abstract}
The Brazilian Federal Constitution of 1988 has assured health as a universal citizen right and created a national health care system - SUS. Nowadays, the Brazilian society has an advanced legal construction assuring social protection trough universal social policies, but a very precarious health care services network.

The aim of this paper is to analyze data from a research in public hospitals in Rio de Janeiro, using different qualitative techniques in order to identify the perceived factors that determine the existence of avoidable inequalities in the access and health services utilization.

The results sign the poor material conditions of health services, associated with discriminatory practices as well as the use of personal relationships as a criterion to access the health system as the material forms of the counter right to health care.
\end{abstract}

Keywords: right to health care; health inequality; social discrimination

\section{Introdução}

A partir da Constituição Federal de 1988, os direitos sociais foram inscritos na condição de cidadania sendo assegurados de forma universal pelo Estado. Em resposta ao forte movimento social que associou a luta pela democracia com a bandeira do Direito Universal à Saúde, foi criado o Sistema Único de Saúde- SUS. A originalidade do projeto do Estado de Bem-Estar Social brasileiro deveu-se à criação de formas de gestão compartilhada e de controle social por parte da sociedade civil e de arenas de pactuação das relações interinstitucionais entre os três níveis governamentais. Além disso, buscou conjugar a existência de sistemas públicos universais de proteção social, organizados como um modelo de seguridade social abrangente, com programas e políticas direcionados para a promoção e inclusão dos grupos mais vulneráveis.
A efetivação do direito à atenção integral à saúde passa, assim, a depender do processo de institucionalização do SUS, em termos legais e normativos e com relação à construção dos sistemas locais de saúde. E o exercício do direito à saúde condiciona-se à capacidade das unidades de saúde de assegurar a qualidade do atendimento e a satisfação das necessidades dos usuários.

Apesar do enorme avanço representado por este desenho constitucional para as políticas sociais nessas duas décadas de sua vigência, essas políticas têm sofrido inúmeras contingências. A institucionalização dos sistemas universais e das políticas voltadas para a inclusão social teve que se enfrentar com um ambiente macroeconômico de ajuste fiscal e estabilização monetária que implicou contenção dos recursos financeiros destinados às políticas sociais, em especial com relação às políticas universais. Essas restrições financeiras, em um momento de expansão dos direitos sociais, implicaram deterioração dos recursos 
materiais e humanos existentes na rede pública e na falta de novos investimentos, imprescindíveis para assegurar a exigibilidade dos direitos e a promoção da inclusão social. Tal situação afetou negativamente a base material como também a cultura institucional nas instituições públicas.

A sociedade brasileira convive hoje com uma avançada construção legal que assegura o bem-estar da população por meio de políticas inclusivas e universais, ao lado de uma institucionalidade precária que não garante o acesso ou a utilização de serviços de qualidade na medida das necessidades da cidadania.

O objetivo deste trabalho é analisar os dados de pesquisa qualitativa realizada em hospitais públicos do município do Rio de Janeiro com usuários, profissionais e gestores a fim de identificar se existem, na percepção desses agentes, condições que impossibilitem o exercício do direito e/ou fatores que favoreçam a existência de desigualdades injustas no acesso e utilização dos serviços de saúde em tais estabelecimentos.

\section{Determinantes sociais de desigualdades injustas}

A preocupação com a equidade em saúde é fundada no princípio ético da justiça distributiva e cada vez mais associada à consideração do direito à saúde como parte dos direitos humanos (Braveman, 2006). A discussão acerca dos determinantes sociais da saúde é constitutiva do campo da medicina social ou saúde coletiva, pois, desde o século XIX, os precursores desse campo já demonstraram a forte relação existente entre as condições sociais em que as pessoas vivem e sua saúde. Essa trajetória contou com iniciativas institucionais como a própria criação da Organização Mundial da Saúde em meados do século XX e os programas de medicina comunitária desenvolvidos nas duas décadas seguintes, que culminaram com o compromisso assumido na Assembleia da OMS de 1976 de alcançar "Saúde para todos no ano 2000". No ano seguinte, a Conferência de Alma-Ata assumia que o meio para realizar esse propósito era a adoção da estratégia de Atenção Primária em Saúde (APS). No entanto, essa estratégia de atenção à saúde deveria se articular dentro de uma abordagem abrangente, que tivesse em conta as causas sociais, econômicas e políticas dos problemas de saúde.

A ênfase nos determinantes sociais da saúde foi arrefecida nos anos posteriores à Declaração de Alma-Ata, tanto pelo desvirtuamento da redução proposta da APS em uma atenção de baixo custo e baixa eficácia quanto pela avassaladora reviravolta dos anos $1980 \mathrm{e}$ 1990, em que o predomínio do neoliberalismo reduziu a ação pública à provisão focalizada de pacotes de atenção básica para os mais vulneráveis. A revalorização dos determinantes sociais da saúde começa a partir da constatação do fracasso das políticas focalizadas em reduzir a pobreza e as desigualdades.

O lançamento das Metas de Desenvolvimento do Milênio em 2000 inaugura essa retomada que se consolida com a criação da Comissão sobre Determinantes Sociais da Saúde- CSDH pela OMS. Com base em sólidos trabalhos de autores como Whitehead (1992) e Marmot e Wilkinson (2003), a CSDH lança o desafio de superar as iniquidades em saúde em uma geração, e o fundamenta:

Social justice is a matter of life and death. It affects the way people live, their consequent chance of illness, and their risk of premature death.

Within countries there are dramatic differences in health that are closely linked with degrees of social disadvantage. Differences of this magnitude, within and between countries, simply should never happen.

These inequities in health, avoidable health inequalities, arise because of the circumstances in which people grow, live, work, and age, and the systems put in place to deal with illness. The conditions in which people live and die are, in turn, shaped by political, social, and economic forces. (Organización Mundial de la Salud, 2008)

Pode-se dizer que a CSDH avançou ao reunir evidências sobre as relações entre as desigualdades em saúde e as desigualdades sociais, posicionando o tema dos determinantes sociais e da equidade no debate mundial sobre a saúde, contudo revelou-se insuficiente no avanço da compreensão da origem dos problemas. Ignorou as relações de poder que moldam os determinantes sociais, associando a responsabilidade das mortes das pessoas às desigualdades, e não aos que se beneficiam das alianças neoliberais que causam as desigualdades (Navarro, 2009), assim como desconsiderou o papel de atores transnacionais, culminando com recomendações políticas abstratas (Arellano, Escudero, \& Carmona, 2008).

Outras lacunas são apontadas em relação ao alcance explicativo dos estudos que buscam reunir evidências e associações entre fatores determinantes e condições de saúde, pois reproduzem as limitações do paradigma dominante na epidemiologia e na saúde pública (Arellano, Escudero, \& Carmona, 2008; Passos, 2009).

Venkatapuram, Bell e Marmot (2010) apontam a relação entre a saúde e os direitos humanos. Consideram que a CSDH demonstra a diferença social que afeta a saúde nos países ricos e pobres como resultado da forma de organização da sociedade através das políticas e práticas econômicas e sociais, mas preocupa-se 
secundariamente com a distribuição social da saúde e da doença, enfocando o controle de doenças e mortalidade por intervenções médicas e o investimento à saúde para o crescimento econômico.

Para os autores, melhorar a saúde e a equidade na saúde como uma questão de justiça social implica a promoção, proteção e cumprimento dos direitos humanos, incluindo o direito à saúde. E, nesse sentido, um papel mais central dos direitos humanos, com vistas à integração das suas análises com a epidemiologia social e medicina social, faz-se necessário. Um dos caminhos propostos é a construção de pontes de análises das causas e distribuição dos problemas de saúde e mortalidade, com o raciocínio ético sobre a justiça social. A partir desse raciocínio ético, como a consideração de que as desigualdades injustas em saúde podem ser evitáveis através de meios razoáveis, podem surgir argumentos para o avanço dos direitos e outros tipos de ação social (Venkatapuram, Bell, \& Marmot, 2010).

No Brasil, foi também criada uma Comissão Nacional de Determinantes Sociais da Saúde- CNDSS, cujo relatório apontou a persistência de inúmeras iniquidades em saúde, com base em uma sólida produção acadêmica. As evidências apontam que as chances de utilização dos serviços de saúde estão fortemente associadas a variáveis sócio-econômicas, tais como raça, anos de estudo, ocupação, acesso a serviços públicos (Neri \& Soares, 2002). Comparações entre pesquisas de amostra domiciliar ocorridas em 1998 e 2003 demonstram a persistência de desigualdades sociais no acesso aos serviços de saúde, sendo a renda o maior fator explicativo da restrição ao acesso. No entanto, existem padrões regionais diferenciados de desigualdades, sendo que no Nordeste este se associa com a renda e escolaridade, enquanto no Sul a relação mais forte se dá com a escolaridade (Travassos, Oliveira, \& Viacava, 2006). Diagnósticos nacionais sobre situação de educação, saúde, renda e emprego apresentam fortes iniquidades em termos de gênero e raça. Eles indicam a situação de inferioridade de negros e, em especial, das mulheres negras, cujo baixo nível educacional resulta em enorme desigualdade em relação ao emprego e renda, como afirma estudo do Instituto de Pesquisa Econômica Aplicada (2008): em 2007, enquanto as mulheres brancas ganhavam, em média, $62,3 \%$ do que recebiam os homens brancos, as mulheres negras ganhavam $67 \%$ do que recebiam os homens negros e apenas $34 \%$ do que recebiam os homens brancos.

Em um survey sobre discriminação racial e preconceito no Brasil, foi encontrada uma tendência à redução do preconceito, em especial dos graus de preconceitos forte e médio, sendo os mais baixos graus encontrados entre os jovens (Venturi \& Bokani, 2004). Esses dados mostram a importância da abertura do de- bate sobre o tema, embora essa retórica esteja distante da realidade retratada nos indicadores socioeconômicos.

Outros estudos sobre o acesso e utilização dos serviços de saúde demonstram que as mulheres que se identificavam como pretas ou pardas tiveram menos acesso a exames de Papanicolau (Amorim, Barros, Cesar, Carandina, \& Goldbaum, 2006) bem como à assistência ao pré-parto e ao parto (Leal, Gama, \& Cunha, 2005). Com relação à utilização, esse estudo mostra que as mulheres negras tiveram menos acesso à escolha $\mathrm{e}$ aos serviços de qualidade e também receberam menos anestesia durante o parto.

Alguns estudos sobre iniquidades no acesso e utilização dos serviços do SUS introduzem a categoria racismo institucional para explicar essas diferenças, definindo-o como o fracasso coletivo de uma organização para prover um serviço apropriado e profissional para as pessoas por causa de sua cor, cultura ou origem étnica (Kalckman, Santos, Batista, \& Cruz, 2007).

Enquanto jovens negros relatam vivências de discriminação em espaços públicos (Cecchetto \& Monteiro, 2006), a identidade sexual também é apontada como fator de discriminação, representando um obstáculo ao acesso aos serviços de saúde (Lacerda, Pereira, \& Camino, 2002).

A importância da variável oferta dos serviços de saúde na produção dessas associações foi pesquisada por Castro, Silva e Vicentin (2005) com relação às internações hospitalares. O estudo conclui que 97 a $99 \%$ da variação na chance de internação são explicadas por características do indivíduo, sendo que somente 1 a $3 \%$ da variação do uso das internações pode ser atribuído a diferenças na oferta.

Esses dados nos levam a formular o problema de nossa investigação em termos das condições e práticas que, mesmo na existência de sistemas públicos universais, reproduzem nos serviços condições diferenciais de acesso e utilização de bens públicos, gerando injustiças e negação dos direitos de cidadania.

\section{Método}

A maior parte dos estudos sobre a interação entre o usuário e os serviços de saúde situa-se no campo da epidemiologia e utiliza os modelos analíticos de acesso e utilização mais conhecidos. O acesso apresenta-se como uma categoria privilegiada de análise, pois é na unidade de relação do usuário com os serviços de saúde que se expressa concretamente o direito à saúde no cotidiano das pessoas (Giovanella \& Fleury, 1996). Os estudos sobre o tema utilizam comumente a conceituação de acessibilidade formulada por Frenk (1985, citado por Giovanella \& Fleury, 1996), a partir de Donabedian, associando-o à disponibilidade de recursos de atenção 
à saúde em determinado local e tempo, bem como as características do recurso que facilitam ou dificultam o seu uso por parte dos clientes potenciais.

Um clássico na análise da utilização dos serviços de saúde é o modelo comportamental de Andersen e Newman, que identifica os fatores explicativos para a utilização de serviços de saúde: os fatores predisponentes correspondem a um conjunto de variáveis demográficas, socioculturais, cognitivas e de atitudes postuladas como fatores pessoais que identificam o grupo de risco potencial, mas não podem ser modificadas por meio de políticas de saúde; os fatores capacitantes consistem nos fatores organizacionais e financeiros que afetam a capacidade do indivíduo para acessar um dado serviço; e as necessidades de saúde representam a percepção individual da necessidade de cuidado ou o diagnóstico por profissionais de saúde (Fleury, 2000; Travassos \& Castro, 2008; Travassos \& Martins, 2004).

No estudo que desenvolvemos procuramos dar ênfase às desigualdades injustas no acesso e utilização dos serviços públicos de saúde, buscando identificar a ocorrência do fenômeno da discriminação, a fim de contribuir para uma análise mais voltada ao enfoque do direito à saúde, para além da abordagem epidemiológica, comum nos estudos sobre o acesso e utilização. É, portanto, no campo dos direitos fundamentais à saúde que se insere a nossa linha de investigação, buscando identificar os fatores percebidos como condicionadores de desigualdades injustas no acesso e utilização dos serviços de saúde.

Tomamos como referência estudos sobre o exercício do direito à saúde e os estudos de Foucault (1977) nos quais afirma que, enquanto a ordem política cria laços jurídicos igualitários entre os cidadãos, as disciplinas operam um contradireito, pois produzem, por meio de laços "privados" entre os indivíduos, assimetrias insuperáveis. Ao contrário de uma contradição, Foucault (1977) vê nessa convivência paradoxal uma imprescindível complementaridade:

A forma jurídica geral que garantia um sistema de direitos em princípios igualitários era sustentada por esses mecanismos miúdos, cotidianos e físicos, por todos esses sistemas de micropoder essencialmente inigualitários e assimétricos que constituem as disciplinas. E se, de uma maneira formal, o regime representativo permite que direta ou indiretamente, com ou sem revezamento, a vontade de todos forme a instância fundamental da soberania, as disciplinas dão, na base, garantia da submissão das forças e dos corpos. As disciplinas reais e corporais constituíram o subsolo das liberdades formais e jurídicas. (p. 195)

Portanto, nossa análise dos processos de discriminação e negação dos direitos da cidadania será feita à luz dos seguintes pressupostos teóricos: a) Os agentes sociais são distribuídos no espaço social geral, na primeira dimensão, de acordo com o volume total de capital que eles possuem e, em segunda dimensão, de acordo com a estrutura de seu capital, isto é, o peso relativo das diferentes espécies de capital, econômico e cultural, no volume total de seus ativos (Bourdieu, 1989).

b) O conceito de Sartre de "serialidade" pode ser útil para teorizar sobre o posicionamento estrutural que condiciona as possibilidades de agentes sociais sem que com isto constitua suas identidades. Posições na estrutura social - de classe, gênero, raça, e idade - condicionam de forma sequencial as vidas dos indivíduos ao viabilizar ou constranger suas possibilidades de ação, e assim conformar relações de superioridade (Young, 2000).

c) A particularidade nas formas de desrespeito, como as existentes na privação de direitos ou na exclusão social, não representa somente a limitação violenta da autonomia pessoal, mas também sua associação com o sentimento de não possuir o status de um parceiro da interação com igual valor, moralmente em pé de igualdade. Para o indivíduo, a denegação de pretensões jurídicas socialmente vigentes significa ser lesado na expectativa intersubjetiva de ser reconhecido como sujeito capaz de formar juízo moral; nesse sentido, de maneira típica, vai de par com a experiência da privação de direitos uma perda de auto-respeito, ou seja, uma perda da capacidade de ser referir a si mesmo como parceiro em pé de igualdade na interação com todos os próximos (Honneth, 2003, pp. 216-217).

Assim, para verificar a ocorrência da percepção de negação do direito à saúde e existência de desigualdades injustas no acesso e utilização dos serviços públicos de saúde, bem como analisar a lógica que orienta os atores envolvidos nestas relações, foi formulado um sistema de hipóteses centrado sobre os seguintes eixos: Formação Profissional, Cultura Institucional, Gestão e Usuários. As hipóteses gerais de cada eixo foram:

1) A formação profissional atribui poder aos profissionais, o que implica hierarquização: (a) no interior das equipes e (b) no atendimento.

2) A cultura institucional não privilegia a noção de atendimento como bem público e exercício de direitos.

3) As condições de gestão de serviços afetam as condições de atendimento.

4) As características dos usuários e dos profissionais confrontadas na dinâmica de uma determinada relação social interferem nas condições de atendimento. 
Essas hipóteses e suas derivadas em cada eixo foram submetidas e reformuladas a partir de uma consulta a informantes qualificados ${ }^{1}$, que também discutiram a seleção dos cinco hospitais públicos, um municipal e quatro federais, onde foi desenvolvido o estudo: Hospital da Lagoa, Hospital do Andaraí, Hospital Geral de Bonsucesso, Hospital Municipal Miguel Couto, Hospital dos Servidores do Estado. A escolha dos mesmos ocorreu em função das localizações geográficas, pois esse é um fator a ser considerado na análise da desigualdade no acesso/utilização dos serviços de saúde; e a opção por hospitais públicos devido ao pressuposto de que o direito à saúde não pode ser negado ou sofrer discriminação em uma unidade estatal responsável pela provisão de um bem público.

A entrada nos hospitais para realização das entrevistas foi precedida pela aprovação dos seus respectivos conselhos de ética e a pesquisa desenvolveu-se de acordo com os padrões prescritos pela Resolução 196/96 do Conselho Nacional de Saúde, concernentes ao envolvimento de seres humanos.

Para estudar a efetivação do direito à saúde e a sua negação através de práticas de discriminação social no atendimento hospitalar, utilizamos metodologia qualitativa com base, inicialmente, em observação participante nas emergências, setores médicos de média e alta complexidade e setores não médicos dos hospitais estudados com base em um roteiro de observação contendo indicadores de acesso/utilização dos serviços de saúde. Através das observações, registradas em diário de campo, foi possível uma aproximação da realidade vivenciada nos hospitais e o ajuste dos roteiros das entrevistas. Posteriormente, realizamos 285 entrevistas semiestruturadas, estando assim distribuídas: 190 com os usuários (e/ou acompanhantes) dos serviços de saúde, com diferentes características e perfis, $90 \mathrm{com}$ os profissionais envolvidos diretamente/ indiretamente com o cuidado em saúde, e 5 entrevistas com os gestores dos hospitais. A escolha dos sujeitos e o quantitativo ocorreram, respectivamente, em função dos critérios de acessibilidade e saturação das respostas, isto é, quando novas entrevistas já não trazem material adicional. Outras duas técnicas de pesquisa, realizadas fora do contexto hospitalar, foram adicionadas, sendo uma delas a promoção de um grupo focal com mulheres negras que atuam como lideranças comunitárias com interface com a área de saúde. A partir dessa técnica, foi possível apreender, através das falas e experiências, a frequência e as situações de discriminação vivenciadas pelas participantes nos serviços de saúde, seus tipos e lógicas, bem como as formas de reações a estas situações percebidas como discriminadoras. A outra técnica consistiu em uma oficina de dramatização - com o método do Teatro do Oprimido, desenvolvido por Boal (2005) - envolvendo um grupo diversificado de profissionais que atuavam como internos em hospitais públicos, não necessariamente os estudados. Através da dramatização eles puderam expressar suas vivências de discriminação, não facilmente captadas com outros instrumentos que apelam mais à racionalidade que às emoções.

O estudo sobre discriminação envolve aspectos culturais que dificultam a expressão de suas vivências. Alguns autores (Abric, citado por Menin, 2006) denominaram zona muda a estes elementos da representação social que não são verbalizáveis e apreendidos pelos métodos tradicionais de pesquisa. Por essa razão, foi necessário combinar diferentes técnicas de investigação de natureza qualitativa.

\section{Resultados}

Os discursos foram agrupados segundo o ator que o enuncia e alocado em categorias relativas aos grandes eixos - formação profissional, cultura institucional, gestão dos serviços e características dos usuários. Apresentaremos aqui os resultados finais do cotejamento dos discursos de diferentes atores nas várias situações estudadas.

\section{Formação profissional}

Os resultados indicam que a instituição hospitalar é altamente hierarquizada, com especial destaque para a posição do profissional médico na cúspide da hierarquia. As equipes profissionais ressentem-se dessa hierarquização, sendo que os níveis mais subalternos, como os profissionais de enfermaria, sentem-se ao mesmo tempo sobrecarregados e desvalorizados, reclamando da falta de reconhecimento do seu trabalho. Os profissionais também sentem que a hierarquização afeta o seu trabalho na medida em que os mais bem situados na hierarquia podem passar aos que estão abaixo os casos que são indesejados. A hierarquização também se dá a partir de variáveis como idade ou tempo de exercício do trabalho na instituição.

Para os usuários, essa hierarquização não parece incomodar, na medida em que compartilham valores comuns em relação à estratificação dos profissionais. No entanto, o uso de uma linguagem inacessível aos pacientes, em especial da parte dos médicos, é vista como uma forma discriminadora de subalternização dos usuários e de discriminação em relação a suas necessidades de reconhecimento.

Diferentemente do pressuposto em nossas hipóteses, não há uma maior proximidade com os usuários dos profissionais situados nos níveis hierárquicos mais baixos e/ou algumas formações mais humanísticas. Ao contrário, os maus tratos e negação dos direitos dos 
usuários por profissionais encarregados da limpeza evidenciam a necessidade destes se colocarem de forma superior, aproximando-se assim aos profissionais.

Algumas formações profissionais mostraram-se mais preocupadas com a necessidade de compreender os problemas dos usuários e suas linguagens. Por um lado, os enfermeiros demonstraram ser essa compreensão uma necessidade da sua prática profissional, embora isso não representasse a capacidade de minimizar os frequentes conflitos com os pacientes. Por outro lado, os assistentes sociais demonstraram uma maior aproximação com os usuários, que se justificaria pelo próprio lugar institucional que ocupa essa categoria. No entanto, essa proximidade na escuta do usuário não foi percebida pelos mesmos como efetiva, já que eles percebem o profissional da área de assistência, na maior parte das vezes, como incapaz de atender a suas demandas.

A posse e utilização de recursos de poder exteriores à relação de atendimento como forma de redução da vulnerabilidade do indivíduo na hierarquia ficou fortemente manifesta em várias formas discursivas, sejam elas o conhecimento do funcionamento do serviço, as relações pessoais com autoridades, o uso de símbolos de poder como jalecos, e até mesmo o recurso último à violência simbólica.

\section{Cultura institucional e gestão}

Formalmente, há um consenso generalizado em relação ao atendimento nos hospitais públicos como direito do cidadão e dever do Estado. No entanto, também é consensual a aceitação de que as precárias condições materiais e de exercício profissional constituem-se em limite justificador da incapacidade de tornar este direito exigível. A naturalização da precariedade nos serviços públicos é compartilhada tanto pelos profissionais quanto pelos usuários, expressando-se concretamente na afirmação: "serviço público é assim mesmo".

A percepção do seu trabalho como uma missão benfeitora é difundida entre os profissionais. Substitui-se dessa maneira a noção formal do dever, inerente à cidadania, pela compaixão, que remete mais propriamente à matriz relacional filantrópica e assistencialista. A compreensão da prática profissional como um favor e da ação estatal como uma benesse contraditam a afirmação do direito à saúde como direito de cidadania. Os próprios usuários enunciam noções contraditórias de direito e de solidariedade, afirmando a boa vontade dos profissionais como um favor. Essa lógica, ao invés de negar a noção de bem público, requalifica-a, entendendo que o SUS é uma atenção pobre para os pobres, e o direito à saúde é um favor da parte dos que atendem.

A inexistência de memória institucional relativa à punição de maus tratos e discriminações por parte da equipe profissional reproduz uma cultura institucional favorável à impunidade, na qual são reforçadas ameaças apenas quando estes atos partem dos usuários em relação aos funcionários. Mesmo a reivindicação dos usuários de que são cidadãos que pagam impostos e devem ser respeitados em seus direitos, utilizada em situações de conflito, é percebida pelos profissionais como um desrespeito.

A aceitação do jeitinho como mecanismo predominante de acesso aos serviços públicos é bastante consensual, entre todos os atores entrevistados. Alguns contrapõem essa prática discriminada à maior transparência nos processos internos, o que reduziria a força deste elemento, que, por não ser igualmente acessível a todos, aumenta as possibilidades de produzir injustiças.

A inexistência de canais de canalização das reivindicações dos usuários, ou seu desconhecimento, aumenta a vulnerabilidade destes às situações discriminadoras. Da mesma forma, a falta de preparo técnico e de formalização de regras no processo de atendimento aumenta o poder discricionário de todos os profissionais que têm contato com o usuário, em especial aqueles envolvidos na triagem.

Com relação à gestão, os dados foram insuficientes para identificar diferentes modalidades de gestão e seu impacto sobre práticas discriminadoras. No entanto, observamos que existe uma surpreendente distância entre o discurso dos gestores e dos demais atores, sejam eles profissionais ou usuários. A ênfase dada pelos gestores, por exemplo, em programas de humanização e promoção profissional, não encontrou ressonância entre os demais discursos.

\section{Características dos usuários}

A existência de características estigmatizantes aumenta fortemente a probabilidade de que o usuário seja discriminado, em especial se elas se apresentarem conjuntamente em um mesmo indivíduo. A menção frequente ao acrônimo PIMBA (pobre, indigente, mendigo, bêbado, atropelado) é prática corriqueira entre os profissionais e denota claramente a rejeição a esse tipo de usuário.

Para alguns gestores e profissionais, a principal forma de discriminação é a condição socioeconômica. No entanto, ela se soma às demais mencionadas, gerando séries de atributos que estigmatizam e provocam atitudes preconceituosas.

Algumas formas de discriminação como o racismo, por serem atualmente mais debatidas, tornam-se mais veladas, dificilmente verbalizadas. No entanto, são explícitas discriminações de gênero, por práticas religiosas de cultos africanos, por orientação sexual divergente da norma, por comportamentos considerados moral ou socialmente condenáveis, tais como praticar o aborto ou engravidar sucessivas vezes, mesmo sendo pobre. A probabilidade de sofrer algum tipo de discri- 
minação aumenta quando se trata de mulher, negra, pobre, vítima de violência. Pacientes com algum tipo de enfermidade que provoca alguma forma de abominação do corpo, tal como odores ou aspecto asqueroso, têm seu atendimento dificultado. Essas formas de discriminação existem desde os profissionais para com os usuários, mas também entre os próprios usuários.

Os usuários têm enorme dificuldade de se colocar no lugar do discriminado. Relatam situações de preconceito e discriminação, mas esse lugar é sempre do outro, nunca de si mesmo. Os preconceitos também se apresentam desde os usuários para com os profissionais, seja por raça, gênero ou idade. Só em condições especiais, quando se trata de lideranças que se organizam para lutar contra a discriminação, é que vamos encontrar um discurso que assume a consciência tanto dos direitos negados quanto das práticas discriminadoras na atenção à saúde. Nesses casos, fica claro que a conscientização e a organização do grupo tornam-se um recurso de poder importante para fazer frente a práticas discriminadoras.

$\mathrm{O}$ fato de serem submetidos a dolorosas peregrinações por várias unidades da rede pública antes de serem atendidos não é percebido como discriminação, embora seja considerado desrespeitoso. Ao contrário, ela tende a ter um efeito inverso, pois quando o acesso é alcançado, o usuário sente-se privilegiado e pouco questiona as condições de sua utilização do sistema.

\section{Conclusão}

A precariedade das condições de funcionamento dos hospitais se torna um dos fatores fundamentais de concretização do contradireito à saúde. A banalização das injustiças provocadas pela inexistência das condições necessárias ao funcionamento em padrões de qualidade torna os profissionais e o usuário reféns da precariedade, o que se expressa no caso do usuário no sofrimento das peregrinações de uma a outra unidade e no caso dos profissionais, na impossibilidade de atender à demanda com qualidade.

O sentimento de ser desrespeitado na sua busca por atenção tem duas consequências cruciais para a negação da cidadania. Por um lado, o desrespeito e a negação do direito ao acesso impedem que o indivíduo se sinta partícipe de uma comunidade política de iguais. Por outro lado, transmuta o exercício do direito em favorecimento.

Outro fator determinante da persistência de desigualdades injustas na atenção hospitalar é a cultura prevalecente que considera as relações pessoais como critério de acesso aos serviços públicos, como mencionado pelos entrevistados: o QI (quem indica). Estudiosa da cultura política brasileira, Barbosa (2006) aponta o jeitinho como expressão na qual a aceitação generali- zada da preponderância da lógica relacional sobre os critérios formalmente definidos de acesso impede que as regras formais sejam a garantia necessária da igualdade requerida pela cidadania (Da Mata, 1983).

Isso tem como consequência impedir que o processo decisório seja transparente, o que acarreta danos às praticas administrativas e, por outro lado, reforça $o$ poder discricionário dos profissionais e atendentes que estão envolvidos com a decisão de negar ou aceitar o paciente. A inexistência de transparência e a inobservância de regras formais partilhadas pelos dois grupos fundamentais, dos profissionais e dos usuários, tornam o acesso um beneficio a ser alcançado na dependência da sorte, das relações pessoais ou até mesmo do uso de recurso ao enfrentamento pessoal.

O aumento do poder discricionário daqueles que selecionam os pacientes a serem atendidos, além de personalizar relações que seriam de direito, permite que a seleção seja feita em base em critérios não formalmente definidos, o que aumenta a possibilidade de discriminação.

Por fim, isto gera um profundo sentimento de insegurança por parte dos usuários em relação à atenção à saúde. Ao contrário da noção de direito social que se fundamenta em uma relação de segurança garantida pelo poder público, o contradireito à saúde está imerso no sentimento de uma profunda insegurança e desamparo. Isto não quer dizer que a atenção hospitalar no SUS não se realize de forma eficaz e efetiva, mas não se materializa como um direito igualmente distribuído à cidadania.

\section{Notas}

Agradecimentos à Valéria Bicudo pela Coordenação do trabalho de campo; à Criola pelo apoio institucional e participação no trabalho campo; à Gabriela Rangel, Tássia Rabelo e Valéria Bicudo pela participação no relatório final. Apoio CNPq e Ministério da Saúde.

1 Especialistas e estudiosos com experiência na área da saúde pública e serviços hospitalares de saúde

\section{Referências}

Amorim, V. M. S. L., Barros, M. B. A., Cesar, C. L. G., Carandina, L., \& Goldbaum, M. (2006). Fatores associados a não realização do exame de papanicolaou: Um estudo de base populacional no município de Campinas, São Paulo, Brasil. Cadernos de Saúde Pública, 22(11), 2329-2338.

Arellano, O. L., Escudero, J. C., \& Carmona, L. D. (2008). Los determinantes sociales de la salud. Una perspectiva desde el taller latinoamericano de determinantes sociales de la salud, ALAMES. Medicina Social, 3(4), 323-335.

Barbosa, L. (2006). O jeitinho brasileiro. Rio de Janeiro: Editora Campus.

Boal, A. (2005). Teatro do oprimido e outras poéticas politicas. Rio de Janeiro: Civilização Brasileira. 
Bourdieu, P. (1989). Social space and symbolic power. Sociological Theory, 7(1), 14-25.

Braveman, P. (2006). Health disparities and health equity: Concepts and measurement. Annual Review Public Health, 27, 167-194.

Castro, H. A., Silva, C. G., \& Vicentin, G. (2005). Estudo das internações hospitalares por pneumoconioses no Brasil, 19842003. Revista Brasileira de Epidemiologia, 8(2), 150-160.

Cecchetto, F. \& Monteiro, S. (2006). Discriminação, cor e intervenção social entre jovens na cidade do Rio de Janeiro (RJ, Brasil): a perspectiva masculina. Revista Estudos Feministas, 14(1), 199-218.

Da Matta, R. (1983). Carnavais, malandros e heróis. Rio de Janeiro: Zahar.

Fleury, S. (2000). Reforming health care in Latin America: Challenges and options. In S. Fleury, S. Belmartino, \& E. Baris (Eds.), Reshaping health care in Latin America: A comparative analysis of health care reform in Argentina, Brazil, and Mexico (pp. 3-24). Canadá: International Development Research Centre.

Foucault, M. (1977). Vigiar e punir: história da violência nas prisões. Rio de Janeiro: Vozes.

Giovanella, L. \& Fleury, S. (1996). Universalidade da atenção à saúde: acesso como categoria de análise. In C. Eibenschutz (Org.), Política de saúde: o público e o privado (pp. 177-198). Rio de Janeiro: Editora Fiocruz.

Honneth, A. (2003). Padrões de reconhecimento intersubjetivo: amor, direito e solidariedade. In A. Honneth, Luta por reconhecimento: a gramática moral dos conflitos sociais (pp. 155-211). São Paulo: Ed. 34. (Obra original publicada 1992)

Instituto de Pesquisa Econômica Aplicada - IPEA. (2008). Retrato das desigualdades de gênero e raça ( $3^{\mathrm{a}}$ ed.). Acesso em 27 de setembro, 2010, em: http://www.ipea.gov.br/sites/000/2/ destaque/Pesquisa_Retrato_das_Desigualdades.pdf

Kalckman, S., Santos, C. G., Batista, L. E., \& Cruz, V. M. (2007). Racismo institucional: um desafio pra a equidade no SUS? Saúde e Sociedade, 16(2), 146-155.

Lacerda, M., Pereira, C., \& Camino, L. (2002). Um estudo sobre as formas de preconceito contra homossexuais na perspectiva das representações sociais. Psicologia: Reflexão e Crítica, 15(1), 165-178.

Leal, M. C., Gama, S. G. N., \& Cunha, C. B. (2005). Desigualdades raciais, sociodemográficas e na assistência ao pré-natal e ao parto, 1999-2001. Revista de Saúde Pública, 39(1), 100-107.

Marmot, M. \& Wilkinson, R. (Eds). (2003). Social determinants of health: the solid facts. Acesso em 14 de maio, 2010, em: http://www.euro.who.int/_data/assets/pdf_file/0005/98438/ e81384.pdf

Menin, M. S. S. (2006). Representação social e estereótipo: a zona muda das representações sociais. Psicologia: Teoria e Pesquisa, 22(1), 43-52.

Navarro, V. (2009). What we mean by social determinants of health. International Journal of Health Services, 9(3), 423-441.
Neri, M. \& Soares, W. (2002). Desigualdade social e saúde no Brasil. Cadernos de Saúde Pública, 18(supl. 1), 77-87.

Organización Mundial de la Salud. (2008). Closing the gap in a generation: Health equity through action on the social determinants of health. Acesso em 14 de maio, 2010, em: http://whqlibdoc.who.int/publications/2008/9789241563703_eng.pdf

Passos, R. (2009). Determinantes, determinação e determinismos sociais. Revista Saúde em Debate, 33(83), 397-406.

Travassos, C. \& Castro, M. S. M. (2008). Determinantes e desigualdades sociais no acesso e utilização de serviços de saúde. In L. Giovanella, S. Escorel, L. V. C. Lobato, J. C. Noronha, \& A. I. Carvalho (Orgs.), Políticas e sistemas de saúde no Brasil (pp. 215-243). Rio de Janeiro: Editora Fiocruz.

Travassos, C. \& Martins, M. (2004). Uma revisão sobre os conceitos de acesso e utilização de serviços de saúde. Cadernos Saúde Pública, 20(supl.2), 190-198.

Travassos, C., Oliveira, E. X. G., \& Viacava, F. (2006). Desigualdades geográficas e sociais no acesso aos serviços de saúde no Brasil: 1998 e 2003. Ciência \& Saúde Coletiva, 11(4), 975-986.

Venkatapuram, S., Bell, R., \& Marmot, M. (2010). O direito de suturas: a epidemiologia social, direitos humanos e justiça social. Health and Human Rights, 12(2), 3-16.

Venturi, G. \& Bokani, V. (2004, agosto/setembro). Sociedade: discriminação racial e preconceito de cor no Brasil. Teoria e Debate Fundação Perseu Abramo, 17(59). Acesso em 27 de setembro, 2010, em: http:/www.fpabramo.org.br/o-que-fazemos/editora/teoria-e-debate/edicoes-anteriores/ sociedade-discriminacao-racial-e-preconceit

Whitehead, M. (1992). The concepts and principles of equity and health. International Journal of Health Services, 22(3), $429-445$.

Young, I. M. (2000). Social difference as a political resource. In I. M. Young, Inclusion and democracy (pp. 81-119). Oxford: Oxford University Press.

Recebido em: 29/09/2010

Revisão em: 27/01/2011

Aceite em: 18/08/2011

Sonia Fleury é Doutora em ciência política, mestre em sociologia e psicóloga. Professora titular da Fundação Getúlio Vargas. Endereço: Praia de Botafogo, 190, sala 518. Rio de Janeiro/RJ, Brasil. CEP 22253-900. Email: sonia.fleury@fgv.br

\section{Como citar:}

Fleury, S. (2011). Desigualdades injustas: o contradireito à saúde. Psicologia \& Sociedade, 23(n. spe.), 45-52. 